\title{
Abstracts for the 16th World Congress for Disaster and Emergency Medicine 12-15 May 2009 Victoria, British Columbia Canada
}

We regret to inform the readers that some errors were identified in some of the abstracts from the 16th World Congress for Disaster and Emergency Medicine in a recently printed issue of Prebospital and Disaster Medicine. The corrected abstracts are printed here.

\author{
Oral Presentation: Spanish \\ Atencion Prehospitalaria en Intoxicaciones con Monoxido de Carbono \\ Guillermo Perez Chagerben \\ Pan-American Trauma Society, Prehospital Care and Urgency Ecuadorian Society, \\ Guayaquil, Ecuador
}

Hoy en día, los incidentes por combustión con materiales tóxicos cada vez ponen en riesgo la contaminación de los bomberos que ingresan a rescatar o combaten en un incendio.

En esta guía enfocaremos el soporte de vida que se brindara a las personas que sufren intoxicaciones con monóxido de carbono y sustancias de mayor concentración en un incendio como el cianuro, principal componente que en el momento de la combustión se desprende, es muy común encontrarlo en objeto de contacto diario.

En la actualidad, existen los riesgos en cada incidente y la seguridad se vuelve un papel principal para el manejo de aquello por eso hay que tener en cuenta los factores predisponentes ante un incendio o una emergencia medicalizada donde la semiotecnia juega un papel importante para la presunción diagnostica del paciente que estaremos atendiendo.

En el año 2000 fue publicado en la revista de la sociedad americana y europea de cirugía torácica que el $50-80 \%$ de las muertes en las salas de UCI o emergencia se debe a inhalaciones por humo en incendios, teniendo en cuenta que en estos países desarrollados el nivel de seguridad es el máximo; minimizando los riesgos del operador o rescatador.

En la clasificación de los humos encontramos los simples, químicos, irritantes, asfixiantes irritantes, tales como; hidrocarburos aromáticos, sulfuro de hidrogeno, humos metálicos.

Esta presentación muestra los resultados del personal médico y paramédico en incidentes con materiales tóxicos (Humo) brindando el soporte prehospitalario hasta el soporte intrahospitalario con la administración de cianocobalamina IV 10,000 mcg endovenosos en $500 \mathrm{ml}$ Solución Glucosada al $5 \%$ o con $500 \mathrm{ml} \mathrm{S}$. S. al $0.9 \%$ prehospitalariamente y preparados intrahospitalarios con diluciones de $10000 \mathrm{mcg}$ en $1,000 \mathrm{ml}$ de S. S. $0.9 \%$ en paciente que presentan un estado moderado de toxicidad.

Prehosp Disast Med 2009;24(3):280

\section{Oral Presentation: Spanish}

Pie de Trinchera en un Inmigrante Arribado en Cayuco a Tenerife

Kevin Garcia Pulido; ${ }^{1}$ Sebastian Matos Castro; ${ }^{1}$

María Pilar Padrón Peña; ${ }^{2}$ Carlos David Rodriguez Martin, ${ }^{1}$

Yurena Garcia Baez; ${ }^{1,2}$ Pedro Dolcet Pérez ${ }^{2}$

1. Servicio de Urgencias Canario, Santa Cruz de Tenerife, Spain

2. Hospital Universitario de Canarias, La Laguna, Spain

Introducción: El frío, es un agente externo que dependiendo de la intensidad y duración de su acción como elemento lesional, puede dar lugar a diferentes síndromes clinicos.

Pie de trinchera: Hace referencia a lesiones localizadas en las extremidades inferiores, producidas por el frío y la humedad durante un periodo de tiempo $(<12 \mathrm{~h})$. 
Término procedente de la Primera Guerra Mundial cuando las tropas permanecían semanas en trincheras mojadas y a baja temperatura.

El pie de trinchera se propicia con la malnutrición, deshidratación, temperatura y tiempo exposición, humedad, ropa inadecuada, mala circulación por calzado inapropiado y la raza.

Caso Clínico: Paciente varón 26 años, inmigrante irregular arribado a nuestras costas procedente del África Subsahariana, tras realizar viaje de 12 días en cayuco.

Presenta deshidratación malestar general y erosiones miembros inferiores, flictenas profundas pierna derecha, desprendimiento de piel en dedos e iniciando necrosis del primer dedo pie derecho.

Pulsos presentes y en eco-doppler no signos de trombosis venosa profunda (sí descenso del flujo arterial)

Evolución: 24 horas después empeoró presentando rabdomiolisis e insuficiencia renal. Apareció fiebre, originalmente se sospechó de origen pulmonar, pero que finalmente fue secundaria a gangrena de la extremidad afectada. Juicio diagnóstico: Pie de trinchera

Tratamiento: Amputación supracondílea de la pierna derecha. Conclusiones: $\mathrm{Al}$ ser un individuo de color, es difícil distinguir los cambios de color propios de la infección. Hay que estar muy pendiente de los cambios de temperatura de los miembros para realizar un tratamiento precoz y eficaz para disminuir la morbilidad y evitar las amputaciones. Prehosp Disast Med 2009;24(3):280-28

\section{Oral Presentation: Spanish}

El Índice de Trauma Pediátrico como Valor Pronóstico en Emergencias Extrahospitalarias

\section{Kevin Garcia Pulido; ${ }^{1}$ Sebastian Matos Castro; ${ }^{1}$}

Maria Pilar Padrón Peña, ${ }^{2}$ Yaiza Prieto Cbico, ${ }^{1}$

Maria Teresa Ramos Fernández;, ${ }^{1}$ Guillermo Burillo Putze ${ }^{2}$

1. Servicio de Urgencias Canario, Puerto de la Cruz, Spain

2. Hospital Universitario de Canarias, La Laguna, Spain

Introducción: El Índice de Traumatismo Pediátrico (ITP) es un instrumento de categorización inicial, rápido, sencillo y objetivo, basado en seis parámetros clínicos y quirúrgicos, aplicable en el lugar del accidente. La puntuación asignada oscila entre 12 y -6. Útil para la categorización del paciente y asignación de recursos, ha demostrado tener un valor pronóstico, pues se distribuye en relación lineal inversa a la mortalidad. Así, con un ITP $<8$, la mortalidad es del $2.5 \%$, $y<0$ pasa al $60 \%$.

Método: Estudio retrospectivo de pacientes pediátricos, diagnosticados de traumatismo, trasladados por una ambulancia medicalizada durante los años 2006 y 2008. Cálculo del ITP extrahospitalario y al llegar al Servicio de Urgencias; evolución y estado al alta hospitalaria.

Resultados: Recogimos 18 casos, edad media 8.3 años, $66.6 \%$ varones. Igual porcentaje por accidentes de tráfico, seguidos de los domésticos (16.6\%). ITP inicial fue 10.61, mientras que en urgencias fue 10.16. No obtuvimos puntuaciones infe- riores a 8. Al alta, el $50 \%$ de los niños presentaba diagnóstico de trauma grave, con secuelas funcionales temporales.

Discusión: No hubo relación entre ITP inicial y diagnóstico al alta, aunque sí entre éste e ITP calculado en urgencias $(p=0.054)$ Al contrario que en los estudios previos (realizados en urgencias hospitalarias) en nuestra serie no hubo relación entre ITP inicial y diagnóstico final.

El escaso número de la muestra puede haber influido en los resultados, por lo que son necesarios otros estudios que incluyan mayor número de pacientes, unidades de asistencia prehospitalaria y hospitales de referencia.

Prebosp Disast Med 2009;24(3):281

\section{Oral Presentation: Spanish}

Tratamiento de la Cardiopatía Isquémica Aguda (CLA) ed Urgencias de Equipos de Atención Primaria (EAP) en el Área Norte de la Isla de Tenerife (Canarias, España)

Kevin Garcia Pulido; ${ }^{1}$ Sebastian Matos Castro; ${ }^{1}$

Aintzane Méndez Urteaga; ${ }^{1}$ Maria Pilar Padrón Peña, ${ }^{2}$ Manuel Gálvez Rodríguez,1,2 Sergio Velazquez Dorta ${ }^{2}$

1. Servicio de Urgencias Canario, Puerto de la Cruz, Spain

2. Hospital Universitario de Canarias, La Laguna, Spain

Introducción: Pese al descenso en los últimos años, la CIA continúa siendo la primera causa de muerte en varones y la segunda en mujeres, con un 12 y un 10\%, respectivamente. Objetivo: Determinar cómo se trata la CIA que acude a los EAP de varias Zonas Básicas de Salud.

Metodología: Estudio descriptivo, realizado en los EAP del Área Norte de Tenerife y una ambulancia de soporte vital avanzado (ASVA) indicativo 43.41.

Analizamos todas las urgencias cardiológicas atendidas en los EAP por una ASVA del servicio de emergencias 11-2 durante seis meses.

Se registra diagnóstico en EAP y en el Hospital de referencia, el tratamiento administrado por el personal de EAP. Registramos si el médico que atendió al paciente había recibido formación MIR o no. Aceptébamos como tratamiento correcto u óptimo si el paciente había recibido oxigenoterapia, aspirina oral, nitroglicerina sublingual o endovenosa y se había canalizado vía venosa.

Resultados: De 48 urgencias cardiológicas, se consideraron procesos isquémicos agudos 44 confirmándose en el hospital 36 (81.8\%) (Diagnósticos definitivos Hospitalarios: 20 IAM, 16 Ángor, 4 Insuficiencia Cardiaca, 4 Fibrilación Auricular, 2 Taquicardia Supraventricular, 2 otros). De estos 36 , recibieron tratamiento adecuado 20 (55.55\%). Los médicos con formación MIR (16) atendieron correctamente al $87.5 \%$ de los casos, mientras que el resto (20) lo hizo en el $30 \%(p=0,023)$. No se observaron diferencias según la zona atendida.

Conclusiones: La atención urgente a la patología isquémica debe mejorarse. Para ello es necesario aumentar la formación de los profesionales que la realizan.

Palabras clave: cardiopatía isquémica; infarto agudo de miocardio; síndrome coronario agudo; tratamiento Prehosp Disast Med 2009;24(3):281 
Oral Presentation: Spanish

Necesidades de Asistencia Urgente a los Inmigrantes Ilegales Recién Llegaods en Cayuco a la Isla de Tenerife (Canaria, España)

Kevin Garcia Pulido; ${ }^{1}$ Sebastian Matos Castro; ${ }^{1}$ Maria Pilar Padrón Peña; ${ }^{2}$ Alberto Matos Padrón; ${ }^{3}$ Manuel Gálvez Rodríguez, ${ }^{2}$ Eduardo Nuñez Molet ${ }^{2}$

1. Servicio de Urgencias Canario, Puerto de la Cruz, Spain

2. Hospital Universitario de Canarias, La Laguna, Spain

Objetivos: Determinar las características de la primera asistencia y las patologías atendidas a inmigrantes que llegan irregularmente vía marítima, en un nuevo tipo de embarcación, el cayuco.

Material y Método: Estudio transversal. Los puntos de asistencia de campaña fueron atendidos por personal del Servicio de Urgencias Canario (SUC), Cruz Roja Española (CRE) y equipo de Atención Primaria (AP) si el número de inmigrantes superaba la capacidad habitual de respuesta. Se incluyeron todos los inmigrantes que llegaron a Tenerife desde el 22 de octubre de 2005 al 21 de octubre de 2006 (1 año). Consignando las patologías diagnosticadas, las derivaciones realizadas y su causa.

Resultados: Llegaron 17.184 inmigrantes. Todos se valoraron en el primer triage, precisando asistencia sanitaria $649(3,7 \%)$. De ellos $447(68,9 \%)$ fueron tratados in situ y 202 trasladados a diferentes centros sanitarios. In situ se trataron 103 hipotermia (23\%), 79 hipoglucemias, 37 erosiones, 21 traumatismos leves, 15 por sarna y 14 dolor abdominal; no se registró el diagnóstico en 16 casos (3.5\%). Los principales diagnósticos de trasladado fueron: 28 deshidrataciones (13.8\%), 22 heridas extremidades, 18 quemaduras, 16 hipotermias, 14 dolores abdominales, 8 hipoglucemias y 8 por vómitos.

Conclusiones: La inmensa mayoria de los inmigrantes llegan en aparente buen estado de salud. De los que precisan atención sanitaria, la tercera parte han de ser trasladados a otros centros. La llegada de inmigrantes irregulares en cayucos requiere un importante despliegue sanitario extrahospitalario, se estima que este fenómeno se seguirá produciendo en los próximos años, y consecuentemente debemos estar preparados.

Palabras claves: Estado de Salud; inmigración; urgencias

extrahospitalarias.

Prehosp Disast Med 2009;24(3):282

\section{Oral Presentation: Spanish}

Satisfaccion Laboral en el Servicio de Urgencias Adultos del Hospital General Regional 25

Rafael A. Chavarria Islas; Elizabeth Sandoval

Asociacion Panamericana de Medicina de Emergencias y Desastres, Mexico, Mexico

Objeticos: Conocer el nivel de satisfacción laboral entre los médicos adscritos al servicio de Urgencias del HGR No 25. Material y Metodos: Estudio Observacional, Prospectivo, transversal y comparativo, incluyó a médicos adscritos al servicio de urgencias adultos del HGZ 25, que fueron encuestados; mediante un cuestionario constituido por 44 items, basado en la escala de actitud tipo Likert constituido por cuatro indicadores: suficiencia, reconocimiento, respeto y confianza. La medición de la actitud fue a través de la siguiente escala: satisfacción muy alta, alta, media, baja y muy baja.

Resultados: Se observa una distribución conforme a la escala de medición de satisfacción laboral, donde 5 médicos se ubican en el nivel bajo, 22 en el nivel medio y 16 en la categoría alta. En la distribución global se observa una distribución en el nivel medio para los tres turnos con un rango de 12.25 a 33 y una mediana de 24 .

Conclusiones: El nivel de satisfacción laboral en nuestro servicio de urgencias se ubica en un nivel medio.

Prebosp Disast Med 2009:24(3):282

\section{Oral Presentation: Spanish \\ Actitudes Docentes de los Medicos de Urgencias del HGR 25}

Jorge Loria Castellanos; Angelica Soria Huerta

Asociacion Panamericana de Medicina de Emergencias y Desastres, Mexico, Mexico

Objetivo: Determinar las actitudes docentes predominantes de los médicos con los que desarrollan su proceso educativo los médicos del servicio de urgencias HGR 25.

Métodos: Estudio observacional autorizado por el comité de investigación. Se incluyeron a los 76 médicos del servicio de urgencias. Se les aplicó un instrumento constituido por 64 items construidos en duplas excluyentes sobre una base de escala tipo Likert de 6 puntos. El instrumento fue validado a través de 2 rondas de expertos en investigación educativa por consenso 4 de 4 . La prueba de KuderRichardson resultó de 0.92. Se empleó estadística descriptiva así como no parametrica (Kruskal-Wallis y $U$ de Mann Whitney). Resultados: La edad media fue de 42.7 años. Predominó el género masculino (56). El (36) correspondieron a médicos del turno matutino. El (73) era personal de base. La especialidad más frecuente fue la de urgencias (41). Solo el (8) tenían formación docente. La antigüedad promedio fue de 13.2 años. El promedio de empleos fue de 2.12. Las actitudes que predominaron fueron los inconsistentes (24) y autoritario (18). Solo hubo diferencias significativas $(p<0.01)$ en las actitudes al comparar la formación docente y el numero de empleos contra las actitudes docentes. A mayor formación docente menos actitudes autoritarias y más democráticas. A menor número de empleos menos actitud inconsistente.

Conclusiones: Es necesario favorecer la formación docente dentro de los médicos del servicio de urgencias a fin de generar actitudes docentes que favorezcan un mejor proceso educativo de los residentes.

Prebosp Disast Med 2009;24(3):282

\section{Oral Presentation: Spanish}

Conducta Terapeutica ante la Hipercalemia en el Servicio de Urgencias

Paul R. Bautista Santos; Javier Ortega Gonzalez

Asociacion Panamericana de Medicina de Emergencias y Desastres, Mexico, Mexico

Objetivo: Determinar la conducta terapéutica en el servicio de urgencias considerando que para determinar el tratamiento anticalemico, es conveniente contar con electrocardiograma y los niveles sericos de potasio; de acuerdo a si 
hay o no calocitosis se normara la conducta terapéutica a seguir. Por lo que con este estudio determinaríamos si hay o no apego a los algoritmos de tratamiento de la hipercalemia. Resultados: Se observo que del total de 132 pacientes atendidos, el $40.09 \%$ era del sexo masculino y $59.09 \%$ femenino; solo al $45 \%$ de los pacientes contaba con electrocardiograma y niveles sericos de potasio como metodos diagnosticos recomendados para tomar la decisión de iniciar la terapia anticalemica adecuada, en el 39\% solo consideraron los niveles sericos de potasio para iniciar la terapia anticalemica y un $16 \%$ se tomo la decisión de iniciar terapia anticalemica utilizando como metodo diagnostico el electrocardiograma. De los electrocardiogramas realizados a los pacientes, el $41.66 \%$ no presento calocitosis, y $58.30 \%$ que presento calocitosis el dato electrocardiografico mas comun fue la presencia de ondas $T$ acuminadas en un $55.3 \%$ seguidos de QRS ancho en un $3.03 \%$. En esta unidad que los fármacos mas utilizados por el personal medico son los siguientes: gluconato de calcio en un $93.18 \%$, furosemide $89.39 \%$, salbutamol spray $59.84 \%$, salbutamol en micronebulizaciones $31.81 \%$, insulina mas dextrosa al $50 \%$ y bicarbonato en un $9.09 \%$. Observamos que solo el $25.75 \%$ del personal medico iniciaba terapia anticalemica con lo establecido en las guias para trata hipercalemia con calocitosis y sin calocitosis; el $74.25 \%$ medicamentos que no se recomendaban en el algoritmo de terapia anticamica.

Conclusiones: No hay apego a los algoritmos de tratamiento anticalemico; es conveniente hacer del conocimiento al personal medico los algoritmos de terapia anticalemica; para proporcionar una atención medica adecuada a los pacientes con IRC e hipercalemia, y evitar el uso inadecuado de medicamentos.

Prehosp Disast Med 2009:24(3):282-283

\section{Oral Presentation: Spanish}

\section{Rasgos Personalidad Predominantes en el Personal} Medico del Servicio Urgencias

Francisco J. Estrada Arreola; Javier Ortega Gonzalez;

\section{Kersty Estrada Alcantara}

Asociacion Panamericana de Medicina de Emergencias y Desastres, Mexico, Mexico

Objetivo: Determinar los rasgos de la personalidad predominantes en el personal medico del servicio de urgencias Métodos: Una vez obtenida la autorización del protocolo por el comité local de investigación, se invitara e informará a todo el personal médico del servicio de urgencias a participar. Posterior a firma de una carta de consentimiento informado se les aplicó el test Psicodiagnostico Gestal de Salama que indican los diferentes bloqueos que pueden presentarse dentro del ciclo de la experiencia y miden los rasgos y actitudes mas frecuentes en el momento presente del individuo. Este test fue evaluado por una psicoterapeuta ajena al estudio.

De acuerdo a los resultados obtenidos de la aplicación del TSG se procedió a realizar el análisis estadístico correspondiente en donde se incluyó estadística descriptiva.
Resultados: E1 43.80\% del personal de urgencias participó. La especialidad que predominó fue la de urgencias (86.95\%), la mayoría eran médicos de base (54.9\%). El 71.8 $\%$ correspondieron al género masculino. Los rasgos de personalidad predominantes fueron retroflexión $(30.43 \%$ ) y retención (26.08\%). Dentro de la de medicina de urgencias y de medicina interna, el rasgo de personalidad predominante fue: retroflexión, en tanto que para medicina familiar fue fijación y retención. Dentro de los residentes de 1er año, el rasgo de personalidad predomínate fue: retención, en tanto que para los de $2^{\circ}$ año fue retroflexión, en los de $3 e r$ año fue postergación y retroflexión y en los médicos de base fue retroflexión.

Conclusiones: Los bloqueos predominantes el los médicos de urgencias son retroflexión y retención. Los rasgos de personalidad predominantes son: manejo de "debeismos" ideas fijas, falta de respeto a los demás y a si mismos. Presencia de remordimiento y resentimientos.

Prebosp Disast Med 2009;24(3):283

\section{Oral Presentation: Spanish \\ Respuesta de los Servicios Medicos de Urgencias ante un Saldo Masivo de Victimas \\ Miguel A. Valladares Aranda \\ Asociacion Panamericana de Medicina de Emergencias y Desastres, Mexico, Mexico}

Ante la presencia de un suceso o un evento en el cual se producen un gran número de víctimas, los sistemas de atención médica de la comunidad son puestos a prueba. De tal forma que deben de estar debidamente estructurados en su accionar, a fin de dar una respuesta oportuna, eficiente y eficaz con la meta común de salvar el mayor número de personas. Es de vital importancia tanto la atención prehospitalaria por los grupos encargados de esta así como los servicios hospitalarios de urgencias que deben responder ambos ante la normatividad y organización de un Centro Regulador de Urgencias Médicas (CRUM) para la adecuada distribución de los lesionados por medio del manejo de protocolos unificados de atención médica de trauma que se guien bajo criterios internacionales como los que marca el TRIAGE con su código internacional de colores (prioridad I color Rojo, Amarillos prioridad II y Verdes prioridad III y Negros prioridad 0 ó cadáveres). Una vez identificados los pacientes con prioridad I se deben de iniciadas las maniobras de atención médica, en la zona de impacto y convertirlos en un paciente adecuado(estabilizado), contar con la ambulancia adecuada para trasladarlo al Hospital o Unidad d Trauma adecuada (Regla de la Triple A). Nace con ello la necesidad de contar en el Sistema una Clasificación de nuestros Hospitales de acuerdo a su capacidad resolutiva siendo los de mayor Capacidad Resolutiva como Hospitales Rojos en donde se deben de recibir todas las víctimas con prioridad I, Hospitales Amarillos con Capacidad Resolutiva Menor y Hospitales Verdes que serán Hospitales de Apoyo para los dos anteriores. Todo lo anterior en el marco legal de la atención de la urgencia médica a nivel prehospitalaria y de los servicios médicos.

Prehosp Disast Med 2009;24(3):283 
Oral Presentation: Spanish

Grupos Medicos de Apoyo Avanzado en Desastre para Evacuacion de Pacientes con Caracteristicas Especiales Miguel A. Valladares Aranda; Felipe Cruz-Vega

Asociacion Panamericana de Medicina de Emergencias y Desastres, Mexico, Mexico

En 2007, el Estado de Tabasco fue víctima de las precipitaciones fluviales que se produjeron en ese año, así mismo la situación geográfica al estar las ciudades en los márgenes de los ríos aumentaba el riesgo de una inundación de consecuencias in comparables. Se activó en forma oportuna y preventiva por medio del monitoreo adecuado de las condiciones hidrometeorológicas que presentaba la región y provocando la toma de decisiones en forma oportuna a fin de que la población en general no sufriera ningún riesgo, lo primero salväritodas las vidas, y continuar la atención médicas : de emergencia. Por tal motivo se decidió evacuar el Hospital más grande de la capital el Hospital General de Zona No 46 del IMSS (ante su eminente inundación), dando prioridad a aquellos paciente en estado crítico garantizando su atención en un área física similar a la que estaban previamente, así mismo se implementaron zonas de atención medicas de urgencias en áreas seguras, con el objetivo de dar continuidad a la atención médica de urgencias y procedimientos quirúrgicos, así como se generaron las medidas de salud pública para evitar brotes de alguna enfermedad y el establecimiento de control de vectores para limitar la aparición de enfermedades como el Dengue entre otras. El Grupo Especial de pacientes lo constituyeron aquellos que se encuentran en el programa institucional de hemodiálisis para lo que se formaron equipos médicos (binomio médico y enfermera) apoyados con equipo electromédico de vanguardia para dar apoyo vital básico y avanzado, utilizando transportación aérea exclusiva, proporcionada por Dependencias del Gobierno Federal, fueron seleccionados debidamente a los pacientes, estabilizados en el área y trasladados con aseguramiento de atención medica en trayecto y llevados a una Unidad Hospitalaria que favorecieran su atención médica integral para someterlos en un programa emergente de hemodiálisis en la Ciudad México. Prebosp Disast Med 2009;24(3):284

\section{Oral Presentation: Spanish}

Papel del Cirujano en Casos de Desastre Giovanni E. Porras Ramirez; Eduardo Vazquez Valdez; Jaime Mier Y Teran; Manuel Peña Vidal

Asociacion Panamericana de Medicina de Emergencias y Desastres, Mexico, Mexico

Que el Cirujano juega un importante papel en estos casos, porque el Cirujano forma parte de un Equipo, que debe conocer el antes, durante, y después del Plan en caso de Desastre Externo (MEA), y del Plan en caso de Desastre Interno (DIE). En el antes, formando parte activa del Centro de Comando, del Área de Triage cerca de la Zona Cero, en el Área de Urgencias o del Ârea de Quirófanos para realizar el Triage Hospitalario, Distribución de Cirujanos y Quirófanos. Conociendo la Disponibilidad y Capacidad de los Quirófanos, Cirujanos y Enfermeras de su Hospital. Conociendo los convenios con otros
Hospitales y participando en los Simulacros anuales. En el durante: Conociendo que un equipo $Q x$. Bien organizado, puede realizar hasta 7 operaciones en 12 horas en circunstancias óptimas, pero en un desastre puede haber factores limitantes por lo que en estos casos puede manejar sólo el $25 \%$ de su capacidad potencial. Y que por lo tanto, el número de lesionados que pueden intervenirse $Q x$ en un lapso de $12 \mathrm{hrs}$ es igual a: Número de Quirófanos x 7/4. Pero también mediante las Tarjetas de funciones para cada elemento del Personal involucrado en estas áreas Que NO todos los Hospitales de Villahermosa Tabasco son Seeguros, porque de 15 hospitales con los que cuenta la ciudad, sólo 4 fueron seguros. Que se necesitan más Médicos ATLS y más Enfermeras con Cursos de Trauma (MIT-E) en los Hospitales Receptores $Y$ que los profesionales de Urgencias en Trauma y Desastres, trabajando hombro con hombro con las Autoridades Gubernamèntales, tenemos la obligación de poner orden y dominar el caos. Prebosp Disast Med 2009;24(3):284

\section{Oral Presentation: Spanish}

Experiencia del Comité Hospitalario para Casos de Desastre del Municipo de Puebla

Ramon Balderas Ruiz; Francisco Sanchez Lopez; Giovanni Porras Ramirez

Asociacion Panamericana de Medicina de Emergencias y Desastres, Mexico, Mexico

El Comité Hospitalario para Casos de Desastre del Municipo de Puebla en el período 2005-2008, con participación de hospitales de los sectores institucional, privado, universitario y militar; teniendo como objetivos: primero, realizar la clasificación de hospitales de acuerdo a su capacidad resolutiva en rojos, amarillos y verdes de acuerdo a criterios de la Academia Mexicana de Cirugía, en segundo lugar; revisar, asesorar y capacitar en los Planes de Emergencia de cada hospital tercer lugar; la implementación, asesoría y supervisión del programa internacional de Hospital Seguro realizando evaluaciones con la cédula de Hospital Seguro que integra la ubicación geográfica, el componente estructural, no estructural y el componente funcional.

Los resultados obtenidos fueron: 1,385 personas capacitadas de 26 hospitales y clínicas del Municipio, 32 juntas del comité. 32 simulacros en total iniciando con la modalidad de simulacro sorpresa a aquellos hospitales que hubieran tenido la experiencia de dos simulacros previos exitosos, tomándolos todos ellos como macrosimulacros por la participación interinstitucional y teniendo como observadores, evaluadores y controladores a personal del comité (representantes de los distintos hospitales, mismo hospital y Protección Civil). Además, se realizó un Magnosimulacro de todo el municipio con la participación de 465,744 personas registradas de 77 dependencias gubernamentales, no gubernamentales, de 2005 edificios con 2,887 brigadistas, 436,397 personas evacuadas hacia puntos de reunión seguros con 178 lesionados simulados, instalando cuatro Puestos de Mando Unificados (PMU) y Centro de Atención y Clasificación de Heridos (CACH) con 4 zona de impacto repartidos en el municipio, la formación del 
Centro Integral de Atención al Desastres (CIAD) en las oficinas de Protección Civil Municipal y la implementación del Centro Operativo para Emergencias Municipales (COEM) en la Presidencia Municipal.

Prehosp Disast Med 2009;24(3):284-285

\section{Oral Presentation: Spanish}

Atencion Prehospitalaria en el Issste

Greta Miranda Cerda; Raymundo P. Hernandez;

Valeria Peralta Leon; Jorge Ordoñez Ceron

Asociacion Panamericana de Medicina de Emergencias y Desastres, Mexico, Mexico

El Instituto de Seguridad y Servicios Socialeș para los Trabajadores del Estado (İSSSTE) ha implementado el servicio de Brigada de Atención de Urgencias desde hace 20 años, su crecimiento ha sido paulatino y la demanda de atención médica de urgencia ha hecho difícil contabilizar la urgencia calificada de la urgencia no calificada en cada área de especialización médica, en virtud de que el facultativo debe estar preparado para enfrentar la situación y solucionarla de la mejor manera posible (1).

El objetivo (2), es alcanzar el desarrollo suficiente para abatir los indices de morbilidad y mortalidad en la población derechohabiente enfermas o lesionadas que requieran ser atendidas y tratadas con oportunidad y eficacia a fin de limitar el daño y tener mayores probabilidades de sobrevivir con el menor número de secuelas posibles.

La valoración de la emergencia "in situ", la clasificación según la gravedad de la misma, el orden de actuación y la preparación para el traslado si el paciente esta fuera de un centro hospitalario son los puntos fundamentales de cuantificar la atención prehospitalaria. La resucitación va a desempeñar un papel importante ante el peligro de muerte y cese de la función de los órganos y sistemas, en que la actuación médica adecuada es fundamental. La atención integral mediante el conjunto de medidas dirigidas de una forma multidisciplinaria esta enfocada a salvar la vida críticamente amenazada (3).

Prebosp Disast Med 2009;24(3):285

\section{Oral Presentation: Psychosocial Issues}

Safety Function Action for Disaster Responders: Innovative Delivery of a Disaster Health Training Program

James Shultz; ${ }^{1}$ Andrea Allen ${ }^{2}$

1. Center for Disaster \& Extreme Event Preparedness (DEEP Center), University of Miami Miller School of Medicine, Miami, Florida USA

2. Barry University, Miami, Florida USA

Introduction: Safety Function Action for Disaster Responders is a disaster health training program for healthcare, public health, and public safety professionals. Features that distinguish this curriculum are: (1) training focused on "disaster health" (a concept introduced in Homeland Security Presidential Directive 21); (2) integration of disaster behavioral health, medical preparedness, and employee health and safety; (3) primary emphasis on practical and psychosocial support for responders; (4) parallel six-strategy framework for use with disaster responders and survivors; (5) applicability throughout all disaster phases; (6) dual training on responder resiliency and survivor psychological support; and (7) structure designed for evaluation.

Methods: Safety Function Action for Disaster Responders is a 20-module curriculum assembled into a five-block "pathway" for ease of training: (1) overview and disaster behavioral health integration; (2) prepare skills set; (3) safety funtion action strategies for Responders; (4) respond skills set; and (5) safety funtion action strategies for Survivors. The same six safety funtion action strategies; safeguard, sustain, comfort, connect, advise, and activate; can be applied by responders for team support and survivor support. During spring 2009, this program is being delivered to 800 hospital, public health, and first responder professionals throughout the State of Florida who agree to serve as training facilitators. DEEP Center staff provides coaching support for these facilitators as they return to their work sites to immediately begin implementing the program with members of their work units. Progress of facilitators and their coworkers along the safety function action "pathway" of modules and activities is tracked and verified.

Results: Preliminary results from the spring 2009 Florida training will be presented in relation to: (1) facilitator evaluations of trainin; (2) documentation of facilitator training at worksites; (3) progress of facilitators and co-workers along the "pathway" activities; and (4) self-reported measures of willingness to serve in a range of emergency and disaster scenarios.

Conclusions: Disaster health training effectively integrates historically separate elements of medical preparedness, public health, and disaster behavioral health.

Keywords: capacity building; education; preparedness; public health; training

Prebosp Disast Med 2009;24(3):s285

\section{Oral Presentation: Psychosocial Issues}

Surge Sort Support: Expanding the Healthcare Highway During Catastrophic Health Events

James Shultz;, ${ }^{1}$ Maurice Ramirez ${ }^{2}$

1. Center for Disaster \& Extreme Event Preparedness (DEEP Center), University of Miami Miller School of Medicine, Miami, Florida USA

\section{High Alert, Kissimmee, Florida USA}

Surge Sort Support is a training program for prehospital and hospital-based healthcare professionals that integrates elements of medical preparedness, public health, and disaster behavioral health.

Surge: During catastrophic health events, the complexity and magnitude of the surge is likely to exceed the numbers of casualties typically included in hospital/health system disaster plans, drills, and simulations. Surge Sort Support extends planning to include medical and psychological casualties, persons searching for missing loved ones who come to hospitals and community care sites, and family members of the casualties. Additional consideration is given to the predictable distress experienced by hospital inpatients, family members of inpatients, and hospital personnel.

Sort: Surge Sort Support introduces a novel triage approach for efficiently processing the multiplicity of casualties. Continuous integrated triage is the only disaster-tested 
approach that fulfills all five criteria of the ideal mass casualty triage system: (1) rapid; (2) scalable; (3) recurring; (4) integrating medical and behavioral triage; and (5) including criteria for exclusion from care. Continuous integrated triage has been field-tested during multiple Disaster Medical Assistance Team (DMAT) hurricane responses. Coupled with the continuous integrated triage model, Surge Sort Support describes the activation and operation of a hospital-based Support Center to process the flow of psychological/behavioral casualties and a hospital-affiliated Family Center to accommodate the "surge of the searching." Surge sort support illustrates how continuous integrated triage in tandem with activation of treatment and support sites effectively "expands the healthcare highway." Using this approach, hospitals are able to handle an influx of disaster survivors and distressed citizens larger in number than the total bed capacity of the facility.

Support: Surge Sort Support adapts the DEEP Center's six-strategy Safety Function Action framework to the support of both hospital staff (building and maintaining resilience for the healthcare workforce) and to patients and citizens served during a catastrophic health event. Safety Funtion Action strategies and tactics can be applied by hospital personnel in all sites serving disaster survivors throughout the triage and treatment process.

Keywords: capacity building; education; preparedness; public health; training

Prehosp Disast Med 2009;24(3):285-286

\section{Poster Presentation: Psychosocial Issues}

Addressing Humanitarian Concerns: Suffering, Service, and Spirituality in the Disaster Setting

Tamasin Ramsay

Social Sciences and Health Research, School of Psychology, Psychiatry and Psychological Medicine, Monash University, Melbourne, Australia

Disasters are not isolated events that happen "to" people, but rather an intricate interplay of political, social, and environmental forces. Anthropology has been instrumental in developing this more complex understanding of disasters. Anthropology offers rigorous methods by which one can study other multifaceted phenomena of human experiences such as suffering, peace, spirituality, and consciousness.

These two areas are addressed in an ethnographic study of the Brahma Kumaris spiritual community in New York and Orissa, India using the qualitative methods of participant observation, discourse analysis, informal discussions, and unstructured interviews.

Data from 12 months of ethnographic research in 2007 and 2008 begin to address important questions such as how does a global spiritual organization find consistent methods of humanitarian service that are sensitive to the traditions and beliefs of the communities in which they serve? After disasters, people with specific technical expertise are required. Is there a place to utilize the technical know-how of spiritual practitioners to respond to the many needs that present themselves to vulnerable populations post-disaster? Is there a place for spiritual preparation for and response to disaster? If so, how can it be done in a way that makes the best use of often misused time, energy, and resources as well as care for those most affected, and without the associated hesitation of spiritual groups? If not, what should spiritual organizations be doing in response to disasters? These critical areas of humanitarian relief will be discussed.

Faith-based non-governmental organizations have an important role in creating resilient communities and providing care in disaster settings. This is an important topic that requires further debate and discussion.

Keywords: disaster; faith-based organization; humanitarian; nongovernmental organization; psychosocial; spirituality Prebosp Disast Med 2009;24(3):s286

\section{Chemical, Biological, Nuclear, Radioactive, and Explosive Educational Stream}

\section{Blast Injuries: Strategic Assessment and Clinical Management}

Carl H. Schultz; ${ }^{1}$ Pinchas Halpern ${ }^{2}$

1. University of California-Irvine, Irvine, California USA

2. Department of Emergency Medicine at Tel Aviv Sourasky Medical Center, Tel Aviv, Israel

Despite the concern regarding nuclear, biological, and chemical terrorism, the most likely weapon terrorists will employ to inflict casualties is a high energy explosive. In the United States alone, $>1,200$ bombings have occurred annually since 1991 . Typical high energy explosives include TNT, C-4, and ammonium nitrate/fuel oil mixtures. Detonation of these compounds produce blast waves capable of generating initial pressures of over $4 \times 106 \mathrm{psi}$. Peak overpressures of as little as 300 psi are capable of dismembering a human body. Exposure to blast energy results in primary, second, tertiary, and quaternary injuries. Primary blast injury results from direct contact with the blast wave and most commonly damages lungs, bowel, and tympanic membranes. Early death frequently results from injury to the lung that includes contusions, alveolar-venous fistulas causing air embolism, hemothorax, pneumothorax, and pulmonary lacerations. The most frequently injured area of the bowel is the colon and these injuries can result in delayed perforation. Secondary blast injuries also are common causes of death and result from objects accelerated by the explosion that strike victims, producing penetrating trauma.

Prehospital management should utilize standard mass casualty triage, resuscitation, and transport protocols. It is helpful to know whether the victim was found inside a structure or outside. Care should be taken to use smaller fluid boluses and be observant for signs of tension pneumothorax. Initial exam in the ED should include evaluating for blunt and penetrating trauma as well as primary blast injury. However, the presence or absence of a perforated tympanic membrane is not a useful marker for serious injuries or patient stability. Protecting the airway requires the modality with the lowest potential for barotrauma that can still maintain the oxygen saturation of at least $90 \%$. If intubation is necessary, maintain tidal volumes at $5-7 \mathrm{ml} / \mathrm{kg}$ and PEEP $<20 \mathrm{~cm} \mathrm{H}_{2} \mathrm{O}$. Shock should be managed in the standard fashion but must include assessment for arterial air embolism. Patients who appear well with normal oxygen saturation after several hours of observation can be sent home. 
Case presentations from the Israeli experience will be presented to highlight some of these concepts.

Prebosp Disast Med 2009;24(3):286-287

\section{Chemical, Biological, Nuclear, Radioactive, and Explosive Educational Stream}

Biological Terrorism: Assessing the Threat, Identifying an Attack, and Providing Clinical Management

Kristi L. Koenig

University of California-Irvine, Irvine, California USA

\section{Objectives}

1. Define the threat of bioterrorism and identify the agents that may be used in biological attacks.

2. Describe the signs and symptoms of biological agent exposure.

3. Discuss the treatment for exposure to various biological agents.

4. Define the basics of immediate patient treatment.

Bioterrorism can be defined as the use of biological agents to kill or sicken people, animals, or plants with the intent to intimidate or coerce a government or civilian population to further political or social objectives. The threat of bioterrorism stems from several elements including: bioweapons are relatively cheap and easy to obtain (though difficult to weaponize); they can induce fear of the unknown; and they have the potential for aerosolized dissemination over a large geographic region so that medical and health resources would be overwhelmed. The US Centers for Disease Control and Prevention has classified biological agents into categories $A, B$, and $C$, with category $\mathrm{A}$ agents considered to be the highest threat. Category A agents are: bacteria (anthrax, plague, and tularemia), viruses (smallpox and viral hemorrhagic fevers like Ebola), and toxins (botulism). These agents are easily disseminated or transmitted from person-to-person (plague, smallpox, and Ebola), have high public health and societal impacts, and have known previous development as biowarfare agents. Healthcare personnel need a high level of suspicion to detect bioterrorism and should have ready access to current diagnostic and treatment information. Key clinical points include: (1) inhalational anthrax patients develop hemorrhagic meningitis in $50 \%$ of cases; (2) secondary pneumonic plague may develop in bubonic plague patients so they need isolation for the first 48 hours; (3) the rash of smallpox spreads from the legs to the trunk and occurs on the palms and soles; all lesions are in the same stage of development; a single case represents terrorism; and (4) botulism (a descending paralysis) should be suspected in patients with concurrent gastrointestinal and neurological symptoms. Public health and law enforcement officials should be involved early for all cases of suspected bioterrorism.

Keywords: anthrax; bioterrorism; bioweapon; botulism; homeland security; plague; smallpox; terrorism Prehosp Disast Med 2009;24(3):287

\section{Chemical, Biological, Nuclear, Radioactive, and Explosive Educational Stream Medical Management of Radiologic and Nuclear Threats \\ Tareg Bey, Kristi L. Koenig \\ University of California-Irvine, Irvine, California USA}

Background: Incidents that involve radionuclear material are uncommon and therefore challenge rescuers and other healthcare providers who may be unfamiliar with medical management of patients with radionuclear contamination. In recent years the possibility of detonation of a radiological dispersal device (RDD) or of a nuclear weapon has led to enhanced efforts for preparedness to manage radiological and nuclear threats.

Discussion: There are numerous radiological sources, for example in hospitals, industry, the military, aerospace, research and the energy sectors. Current terrorist scenarios that involve a radiolologic or nuclear threat include: an RDD (or dirty bomb); nuclear device detonation; meltdown of a nuclear reactor; and placement of radioactive material in public places. Current disaster preparedness focuses on the following radionuclides as the most likely to be used for terrorist activity: Cobalt-60, Cesium-137, Iridium-192, Strontium-90, Radium-226, and Plutonium238. There are three types of radiation exposures: irradiation, external contamination, and internal contamination. For irradiation, time, distance and shielding are the most important protective measures. For external contamination, removal of clothing and cleaning of contaminated wounds is important to prevent further internal contamination. Internal contamination with radioisotopes is difficult to treat and the use of radiological antidotes like Diethylenetriamene pentaacetate (DTPA) is time sensitive and requires extensive medical resources. Many radioisotopes like Polonium-210 lack a radiological antidote and once internalized the radioisotope can lead to death. Patients with major life-threatening trauma associated with radiological contamination pose a special challenge to rescue and other healthcare providers. The key concept is that life-threatening traumatic injuries like exsanguination should be treated first before decontamination. Traumatic injuries can be fatal, whereas healthcare personnel exposure to radiation caused by dust or liquids is minimal, except with strong and compact gamma radionuclides like Cobalt-60.

Summary: Additional education for healthcare providers, rescue and public safety personnel is important. Patients with concurrent major life-threatening trauma and radionuclear contamination should be treated according to standard trauma resuscitation protocols before extensive decontamination is performed.

Keywords: antidode; dirty bomb; nuclear; radioisotope; radiological; radiological dispersal device

Prehosp Disast Med 2009;24(3):287 
Statement of Ownership-Management and Circulation-Required by 39 U.S.C. 3685

1. Publication title: Prehospital and Disaster Medicine

2. Publication number $1049-023 \mathrm{X}$

3. Filing date: October 30,2008

4. Issue frequency: Bi-Monthly

5. Number published annually: 6

6. Annual subscription price: $\$ 120.00$ Individual/ $\$ 400.00$ Institution

7. Mailing address of known office of publications: 3330 University Avenue, Suite 130 (Dane County) Madison, WI 53705-2167 USA

8. Mailing address of the headquarters of the general business office of the publisher: Same.

9. Publisher: World Association for Disaster and Emergency Medicine, 3330 University Avenue, Suite 130 (Dane County) Madison, WI 537052167 USA

10. Owner: World Association for Disaster and Emergency Medicine, 3330 University Avenue, Suite 130 (Dane County) Madison, WI 53705-2167 USA

11. Known: bondholders, mortgages, and other security holders owning or holding 1 percent or more of total amounts of bonds, mortgages or other securities: None.

12. The purpose and function and nonprofit status of this organization and the exempt status for federal income tax purposes have not changed during the preceding 12 months.

Extent and Nature of Circulation:

1. Total copies: 800 -aveg. per issue during proceeding 12 months/1,100 last issue.

2. Paid/Requested Circulation: Outside country mail subscriptions 326

3. Others mailed through USPS 2

4. Free distribution outside the mail 342

5. Copies not immediately distributed 118 (used for education and promotion)

I certify that all information furnished is true and complete.

Marvin L. Birnbaum, MD PhD, Editor-in-Chief and Director of the WADEM Business Office 